\title{
First study of risk factors for REM sleep behaviour disorder uncovers a few surprises
}

REM sleep behaviour disorder (RBD) is a parasomnia involving dream enactment, and is a prediagnostic sign of Parkinson disease (PD) and other synucleinopathies. A recent multicentre study has highlighted key risk factors for this sleep disorder. "Studying patients with RBD provides an unprecedented window into very early stages of neurodegenerative disease," says Ron Postuma, lead author of the study.

Male sex and older age are known risk factors for RBD, but environmental links have been unclear. Postuma et al. conducted a questionnaire-based study in 347 patients with RBD from 13 sleep centres spanning the USA, Canada, Europe and Japan, who were matched with 347 controls. "As RBD is relatively uncommon, only by combining forces did we have the power to assess environmental risk factors," says Postuma.

As for PD, prior head injury and occupational exposure to pesticides were associated with increased risk of RBD. Other risk factors, however, showed divergent relationships with $\mathrm{RBD}$ and PD: whereas caffeine and smoking are strongly protective against $\mathrm{PD}$, they were not protective in the case of RBD. Indeed, the odds ratio for RBD was 1.43 in ever-smokers compared with nonsmokers.

"These findings have important implications for our understanding of the mechanism of caffeine and smoking protection in PD," comments Postuma. "It might suggest that they 'protect' against PD by selectively acting on the substantia nigra-the region responsible for the primary motor manifestations-without affecting the synucleinopathy in general."

The researchers also found that individuals with RBD had fewer years of formal schooling than did controls, and were more likely to have worked as a farmer. These two factors seem to interact, however, as adjusting for education reduced the assocation between RBD and farming to borderline statistical significance. The reason why education status affects RBD risk is currently unclear, but could involve

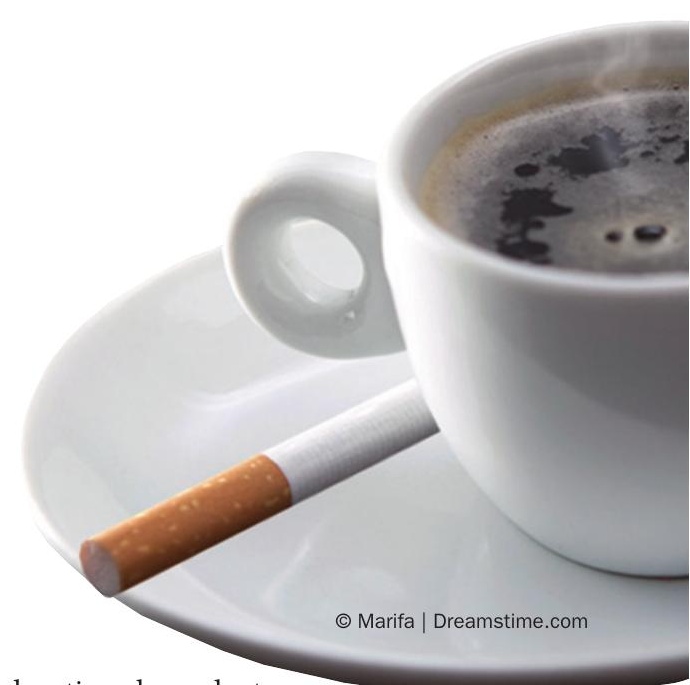

education-dependent

differences in occupation and associated environmental risk factors.

The findings highlight promising avenues for future research. "With time, we will be able to look at the association between these risk factors and the eventual risk of dementia or PD," says Postuma.

Katie Kingwell

Original article Postuma, R. B. et al. Environmental risk factors of REM sleep behavior disorder. A multicenter case-control study. Neurology doi:10.1212/

WNL.0b013e31825dd383 